\title{
Efeito da atividade física sobre densidade mineral óssea de mulheres pós menopausa
}

\section{Effect of physical activity on bone mineral density in postmenopausal women}

\author{
Elias Ferreira Porto ${ }^{1}$ \\ Claudia kümpel ${ }^{2}$ \\ Ilzane da Silva Souza Batista ${ }^{3}$ \\ Eduardo Filoni ${ }^{4}$ \\ Jonatas da Silva Souza ${ }^{5}$ \\ Ana MJ Ferracioli ${ }^{6}$ \\ Antônio Adolfo Mattos de Castro ${ }^{7}$
}

\section{RESUMO}

Introdução: A osteoporose vem sendo um grande problema de saúde pública, tem se tornado uma das principais doenças incapacitantes principalmente de mulheres, por apresentar um menor pico de massa óssea e também pelas consequências provenientes da menopausa. Um importante exame deve ser feito a densitometria óssea, sendo um meio não invasivo para a avaliação de pacientes com osteoporose, possibilitando a realização de seu diagnóstico e seguimento. Objetivo: avaliar se a atividade física regular e no estilo de vida podem influenciar na densidade mineral óssea de mulheres maiores de 40 anos. Método: Estudo transversal com abordagem quantitativa, composto por dois grupos, o Grupo I formado por mulheres fisicamente ativas (25), e o Grupo II foi formado por mulheres sedentárias (25). Todos responderam aos seguintes questionários: Questionário internacional de atividade física versão curta (IPAQ), Escala Perfil de Estilo de Vida Individual (NAHAS), e um questionário elaborado pelos avaliadores com o objetivo de obter informações mais especificas dos avaliados. Foram incluídas mulheres maiores de 40 anos, que realizaram o exame de densitometria óssea, que fazia ou não atividade física. Resultados: Foram avaliadas 50 mulheres sendo que 25 eram ativas fisicamente e 25 sedentárias, a idade média foi de 60,6 $\pm 9,7$ anos, com IMC de 26,5 $\pm 6,1 \mathrm{~kg} /$ $\mathrm{m}^{2} .8 \%$ da amostra eram tabagistas $8 \%$ consumiam bebidas alcoólicas regularmente 33\% eram diabéticas, 30\% era dislipidêmico e 63\% eram hipertensas. A proporção de indivíduos sedentários que dissera que sua saúde era pior em relação a pessoas de sua mesma idade foi significantemente maior do que para o grupo de mulheres ativa fisicamente, isto também foi visto quando perguntado se a sua saúde tinha piorado em relação há um ano anterior $(p=0,003)$ e $(p=0,003)$ respectivamente. 0 estilo de vida foi diferente significantemente entre os grupos $(p=0,002)$ sendo mais

\footnotetext{
${ }^{1}$ Doutor em medicina translacional e professor do programa de mestrado em promoção da saúde do Centro Universitário adventista de São Paulo.

${ }^{2}$ Mestre em geriatria e Professora do curso de fisioterapia do Centro Universitário adventista de São Paulo.

${ }^{3}$ Bacharel em Fisioterapia pelo Centro Universitário adventista de São Paulo.

${ }^{4}$ Doutor em ciências médicas e Professor da Faculdade Inspirar/São Paulo.

${ }^{5}$ Mestre em promoção da saúde e professor do curso de fisioterapia do Centro Universitário adventista de São Paulo.

${ }^{6}$ Bacharel em Fisioterapia pelo Centro Universitário adventista de São Paulo.

${ }^{7}$ Doutor em pneumologia e professor da Universidade federal dos Pampas
} 
positivo para o grupo de indivíduos ativos; assim como, a densidade mineral óssea da colona lombar foi significante maior para o grupo dos indivíduos fisicamente ativos $(\mathrm{p}=0,02)$. 0 T-score e o Z-score foram significantemente mais negativos no seguimento do fêmur total para o grupo sedentário do que no grupo dos ativos fisicamente $(p=0,04)$ e $(p=0,04)$. Conclusão: o grupo de mulheres ativa fisicamente apresentaram estilo de vida mais positivo, maior densidade mineral óssea, consecutivamente menor risco para osteopenia e osteoporose.

\section{PALAVRAS-CHAVE}

Estilo de vida; Atividade física; Osteoporose. 


\section{INTRODUÇÃo}

A população idosa vem crescendo acentuadamente nos países desenvolvidos e naqueles em desenvolvimento decorrente ao aumento na expectativa de vida. Com esse aumento da sobrevida, se eleva a ocorrência de doenças relacionadas ao envelhecimento da mulher. Entre essas doenças destaca-se a osteoporose (BUTTROS., et al 2011).

A osteoporose é uma doença caracterizada pela perda osteometabólica substancial da massa óssea e deterioração da microarquitetura do tecido ósseo afetando a resistência óssea, qualidade e aumentando o risco de fratura que afetam o músculo e o sistema esquelético, causando dor crônica, perda da capacidade funcional e comprometendo a qualidade de vida, e aumentando mortalidade (MOREIRA et al., 2014).

Os fatores de risco mais valorizados que levam a osteoporose: o gênero feminino, a etnia amarela e branca, a idade mais avançada, menopausa precoce, a hereditariedade, história pregressa de fraturas osteoporóticas, nutrição inadequada, maus hábitos (ingestão exagerada de café, álcool, tabaco), sedentarismo, certas medicações (glicocorticoides, anticonvulsivantes) e doenças como a artrite reumatoide e quase todas as doenças inflamatórias sistêmicas (SOUZA et al., 2010).

Essa doença tem maior ocorrência na população feminina, por apresentar um menor pico de massa óssea e também das consequências provenientes da menopausa. Nessa população a prevalência de osteoporose na coluna lombar varia de 15,8\% em mulheres na faixa de 50 a 59 anos a $54,5 \%$ em mulheres com mais de 80 anos, e a prevalência de fraturas varia entre $20 \%$ e $82 \%$, nas mesmas faixas etárias, respectivamente (CAPUTO et al., 2014).

A prática de atividade física ao longo de diferentes fases da vida tem sido sugerida como fator de proteção de osteoporose. De fato, alguns estudos epidemiológicos transversais analisando a influência da prática de atividade física na infância ou na idade adulta têm sugerido que os indivíduos mais ativos fisicamente são aqueles que apresentam maior DMO (KOHRT et al.,1997).

0 presente estudo teve por objetivo avaliar se a atividade física regular e no estilo de vida podem influenciar na densidade mineral óssea de mulheres maiores de 40 anos.

\section{MÉTODOS E MATERIAIS}

Este é um estudo transversal com abordagem quantitativa no qual foram analisados um grupo de mulheres maiores de 40 anos. 0 grupo de mulheres fisicamente ativas foram selecionadas de um grupo de indivíduos que realizam atividades física dentro dos campos da universidade. Já o grupo de mulheres sedentárias foram selecionadas a partir de indicações das próprias mulheres do grupo ativo fisicamente.

Todos os voluntários participantes da pesquisa assinaram o Termo de Consentimento Livre e Esclarecido (TCLE), em duas vias elaborado para atender as propostas da Resolução do Conselho Nacional de Saúde- CNS no 466 do Ministério da Saúde. 0 projeto de pesquisa foi aprovado pelo Comitê de Ética e Pesquisa do Centro Universitário Adventista de São Paulo (CEP/UNAS) sob o número 1.820 .836$.

Todos os participantes responderam aos seguintes questionários que foram aplicados: Questionário internacional de atividade física versão curta (IPAQ), Escala Perfil de Estilo de Vida Individual (NAHAS), e um questionário elaborado pelos avaliadores com o objetivo de obter informações mais especificas dos avaliados. Os dois primeiros questionários são validados para a população brasileira. Todos os pacientes realizaram o exame de densitometria óssea para avaliar a densidade mineral óssea.

A População de estudo foi constituída por $50 \mathrm{mu}$ lheres onde 25 eram ativas física e 25 sedentárias com idade a 40 anos. Os indivíduos envolvidos neste estudo apresentaram os seguintes critérios de inclusão: ser maior de 40 anos do sexo feminino e estar disposto a realizar os exames propostos. Não participaram deste estudo Indivíduos que apresentasse diagnóstico de esquizofrenia ou qualquer condição mental que impedisse a continuidade do trabalho individuo do sexo masculino, e mulheres que fizesse uso de corticoide oral no último ano previamente a pesquisa, recebendo tratamento quimioterápico, ou aquelas que estivesse fazendo reposição hormonal ou uso de vitamina D.

\section{Questionário Internacional de Atividade Física (IPAQ)}

O IPAQ é um instrumento que permite estimar o tempo semanal gasto na realização de atividades físicas 
de intensidade moderada a vigorosa e em diferentes contextos da vida (trabalho, tarefas domésticas, transporte e lazer). Há a possibilidade de estimar o tempo despendido em atividades mais passivas (realizadas na posição sentada). Utilizando-se o IPAQ, obteve-se um escore contínuo expresso como equivalente metabólico por minuto por semana (METmin/semana), tanto por domínio como total. De acordo com a quantidade de MET, o nível de atividade física foi classificado como: baixo (inferior a 600 MET por minuto por semana), moderado (de 600 a 1499 MET por minuto por semana) e alto (igual ou superior a 1500 MET por minuto por semana)19. As pacientes foram assim classificadas, quanto à $\mathrm{AF}$, em sedentárias, ativas ou muito ativas. Foram avaliados, para cada domínio diferente do questionário, os percentuais de pacientes praticantes e o gasto energético, em MET-minuto/semana.

\section{Escala Perfil de Estilo de Vida Individual}

O questionário Escala Perfil de Estilo de Vida Individual que é um instrumento para mensurar estilo de vida baseado nos componentes "Nutrição", "Atividade física”, "Comportamento preventivo", "Relacionamento sociais" e "Controle do estresse" e a versão brasileira da Escala Perfil de Estilo de Vida Individual é um instrumento auto administrado que considera o comportamento dos indivíduos no último mês e cujos resultados permitem determinar a associação entre o estilo de vida e a saúde. 0 questionário tem 14 questões, para cada questão há possibilidade de zero a 3 pontos. Ao final o questionário será somado, se o entrevistado obtiver uma pontuação até 16 pontos é considerado um estilo de vida negativo e necessita de mudança urgente. Pontuação entre 17 e 25 pontos é compatível com um estilo de vida razoável, e acima de 26 pontos é considerado um bom estilo de vida.

\section{Questionário de avaliação de hábitos de vida}

O questionário de avaliação de hábitos de vida é dividido em duas partes; a primeira é referente a dados socioeconômico e cultural do indivíduo, e segunda parte sobre hábitos de vida. Foi utilizado para confirmar se o indivíduo tem ou não um hábito de vida saudável. Foram considerados indivíduos com hábitos de vida posi- tivo aqueles que responderem positivamente a pelo menos $80 \%$ dos requisitos dos oito apresentados no questionário de fatores relacionados ao Habito de Vida, que incluem: quantidade de sono, família, alimentação, atividade física, uso de tabaco e de bebida alcoólica, visita ao médico e estresse.

\section{Densitometria óssea}

O exame de densitometria óssea serve para medir a densidade dos ossos a fim de diagnosticar a osteoporose e avaliar o risco de fraturas. Usam uma tecnologia avançada de Raio-X, conhecida como absormetria radiológica de dupla energia ou DEXA (DualEnergy X-ray Absorptiometry) Cromox ${ }^{\circledR}$ USA, para detectar a osteoporose. 0 DEXA, atualmente, é um método estabelecido para medir-se a densidade mineral óssea (DMO). É um procedimento rápido e indolor para a medição da densidade mineral óssea. Com ele, essa medição é feita na parte inferior da coluna vertebral e nos quadris. Os resultados são apresentados em gramas/cm2 e comparados à média das pessoas de 20 anos de idade (T score), que representa o valor do pico da massa óssea. Também são comparados aos valores médios da DMO das pessoas de mesma idade (Z score). São calculados as porcentagens relativas e os desvios padrões (DPs) das médias. Os resultados são considerados, conforme consenso da OMS, como Normal, quando a densitometria mostra até -1 desvio padrão no T score; Osteopenia, de -1 a -2,5 DPs e Osteoporose de -2,5 DPs para mais. Há ainda a denominação de Osteoporose estabelecida quando, além do DP $<-2,5$, o paciente apresenta uma fratura osteoporótica. Hoje se considera como portador de OP qualquer paciente que tenha tido uma FxOP. O Z score com valores iguais ou menores que - 2 é sugestivo de uma possível Osteoporose secundária.

\section{RESULTADOS}

Foram avaliadas 50 mulheres sendo que 25 eram ativas fisicamente e 25 sedentárias, a idade média foi de $60,6 \pm 9,7$ anos, com IMC de $26,5 \pm 6,1 \mathrm{~kg} / \mathrm{m}^{2} .8 \%$ da amostra eram tabagistas $8 \%$ consumiam bebidas alcoólicas regularmente $33 \%$ era diabética, $30 \%$ era dislipidêmico e 63\% eram hipertensas. A proporção de indivíduos sedentários que dissera que sua saúde era pior em relação 
a pessoas de sua mesma idade foi significantemente maior do que para o grupo de mulheres ativa fisicamente, isto também foi visto quando perguntado se a sua saúde tinha piorado em relação há um ano anterior $(\mathrm{p}=0,003)$ e $(\mathrm{p}=0,003)$ respectivamente. 0 estilo de vida foi diferente significantemente entre os grupos, há maior proporção de mulheres ativas fisicamente que foram consideradas como tendo hábitos de vida positivo, $(\mathrm{p}=0,002)$ tabela 1 .

Tabela 1. Avaliação da densidade mineral óssea em três seguimentos corporais.

\begin{tabular}{|c|c|c|c|}
\hline Variáveis & $\begin{array}{l}\text { Grupo fisicamente ativo } \\
n=25\end{array}$ & $\begin{array}{c}\text { Grupo sedentário } \\
n=25\end{array}$ & $\mathbf{p}$ \\
\hline Idade (anos) & $60,7 \pm 10,3$ & $60,3 \pm 8,4$ & 0,87 \\
\hline IMC $\left(\mathrm{kg} / \mathrm{m}^{2}\right)$ & $26,9 \pm 4,3$ & $27,2 \pm 4,2$ & 0,84 \\
\hline Sexo $(M / F)$ & 25 & 11 & \\
\hline Tabagistas (\%) & 8 & 9 & 0,97 \\
\hline Ex-tabagistas (\%) & 20 & 21 & 0,32 \\
\hline Etilistas (\%) & 4 & 9 & 0,32 \\
\hline Ex-etilista (\%) & 0,12 & 0,18 & 0,26 \\
\hline Horas de sono & $7,1 \pm 1,0$ & $6,9 \pm 1,4$ & 0,45 \\
\hline \multicolumn{4}{|l|}{$\begin{array}{l}\text { Saúde em relação ano } \\
\text { passado }\end{array}$} \\
\hline Pior (\%) & 8 & 36 & $<0,0001$ \\
\hline Igual (\%) & 52 & 45 & 0,32 \\
\hline Melhor (\%) & 40 & 18 & 0,002 \\
\hline \multicolumn{4}{|l|}{$\begin{array}{l}\text { Saúde em relação } \\
\text { indivíduos de mesma } \\
\text { idade }\end{array}$} \\
\hline Pior (\%) & 8 & 36 & $<0,0001$ \\
\hline Igual (\%) & 40 & 27 & 0,09 \\
\hline Melhor (\%) & 52 & 36 & 0,03 \\
\hline Diabéticos (\%) & 32 & 36 & 0,67 \\
\hline Dislipidêmicos (\%) & 28 & 36 & 0,76 \\
\hline HAS (\%) & 64 & 63 & 0,99 \\
\hline Estatinas (\%) & 36 & 45 & 0,34 \\
\hline NAHAS (pontos) & $28,2 \pm 7,1$ & $25,8 \pm 7,1$ & 0,032 \\
\hline $\begin{array}{l}\text { IPAQ (METmin/ } \\
\text { semana) }\end{array}$ & $425,3 \pm 125,4$ & $910,5 \pm 136,5$ & $<0,0001$ \\
\hline
\end{tabular}

A densidade mineral óssea da colona lombar foi significante maior para o grupo dos indivíduos fisicamente ativos, mas não foi encontrada diferença para a densidade no colo do fêmur e de fêmur total, Figura 1. 


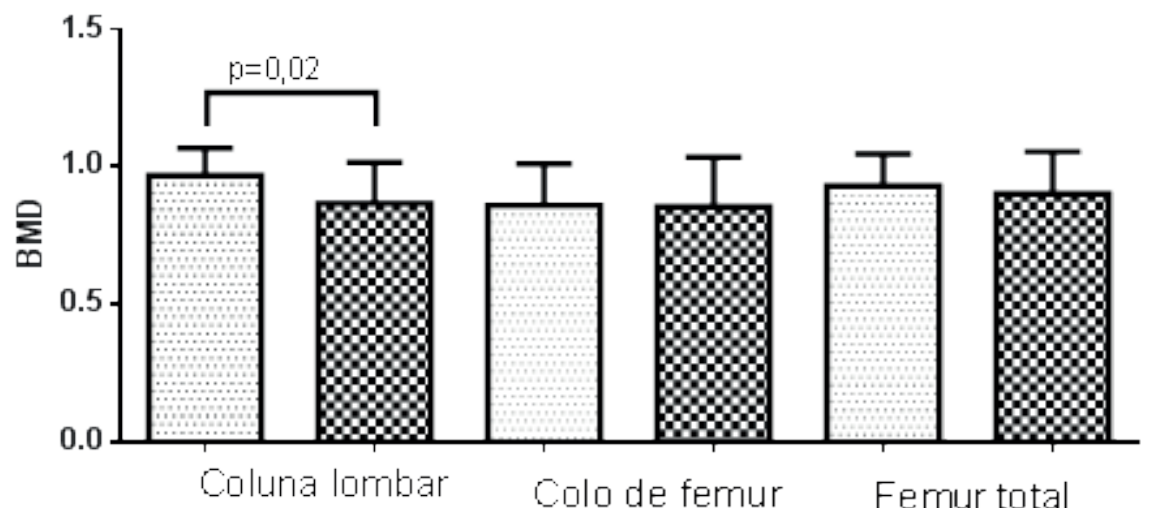

Figura 1. Avaliação da densidade mineral óssea para ambos os grupos.

\section{Avaliação do T-score em três seguimentos corporais}

O T-score foi significantemente mais negativo no seguimento do fêmur total para o grupo sedentário -0,8 $\pm 1,06$ do que no grupo ativo fisicamente $-0,1 \pm 1,2(p=0,04)$. Embora para o seguimento de colo do fêmur não tenha havido diferença significante, para o grupo de sedentários a média também foi menor $-1.1 \pm 1,2$ e $-0,8 \pm 0,9$ e coluna lombar 1,3 $\pm 1,4$ e $-1,1 \pm 1,1$ sedentários e ativos fisicamente respectivamente, figura 2 .

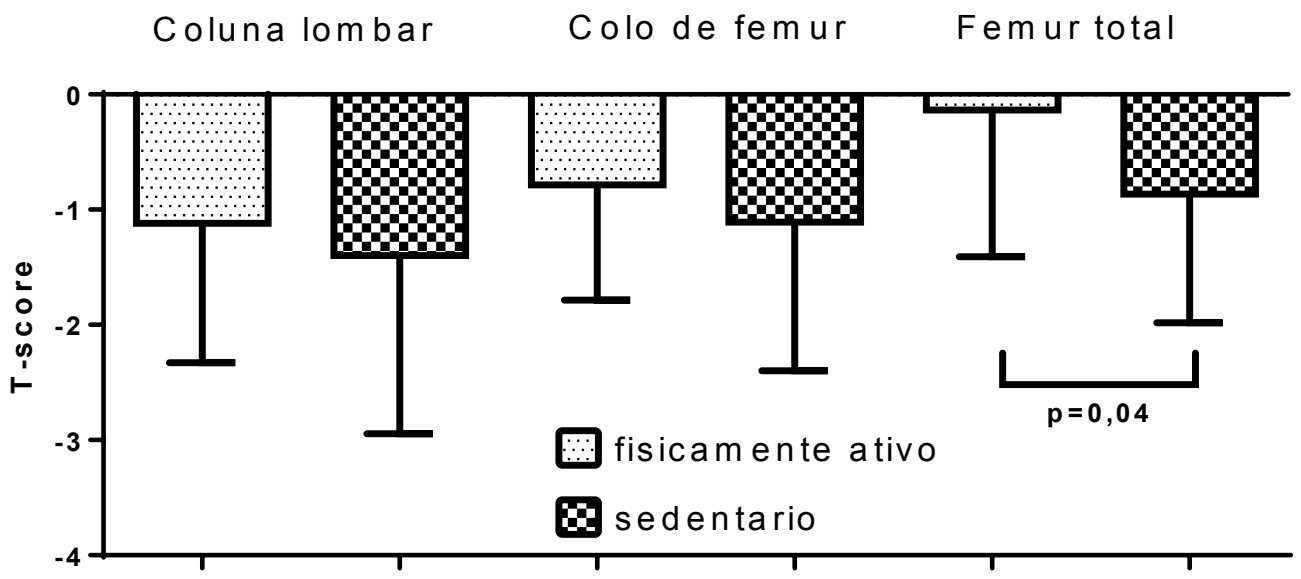

Figura 2. Avaliação do T-score para ambos os grupos.

\section{Avaliação do Z-score em três seguimentos corporais}

0 Z-score foi significantemente menor no seguimento do fêmur total para o grupo sedentário 0,08 $\pm 1,15$ do que no grupo ativo fisicamente $0,7 \pm 1,2(p=0,04)$. Embora para o seguimento de colo do fêmur não tenha havido diferença significante, para o grupo de sedentários a média também foi menor $-0,7 \pm 0,9$ e - $0,44 \pm 1,28$ e coluna lombar $1,3 \pm 1,4$

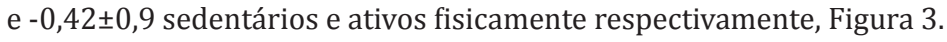




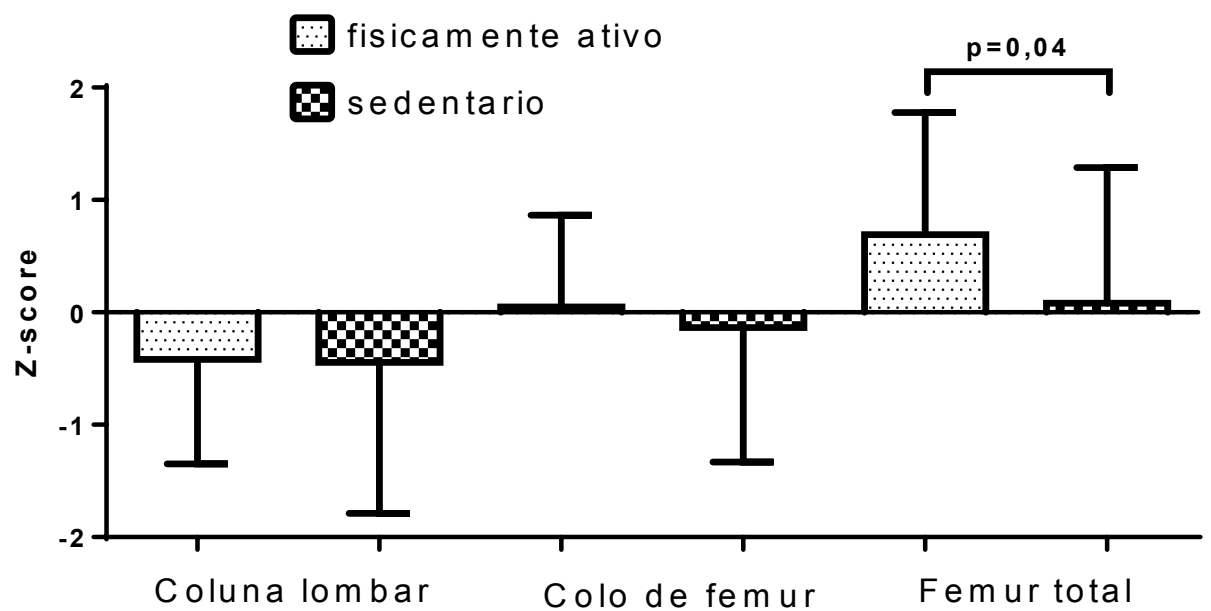

Figura 3. Avaliação do Z-score para os grupos.

\section{Correlação entre T-score e nível de atividade física}

Foi encontrado uma correlação positiva entre o nível de atividade física e o T-score, ou seja quanto maior foi o nível de atividade física que o indivíduo se encontrava, mais positivo foi o t-score, figura 4

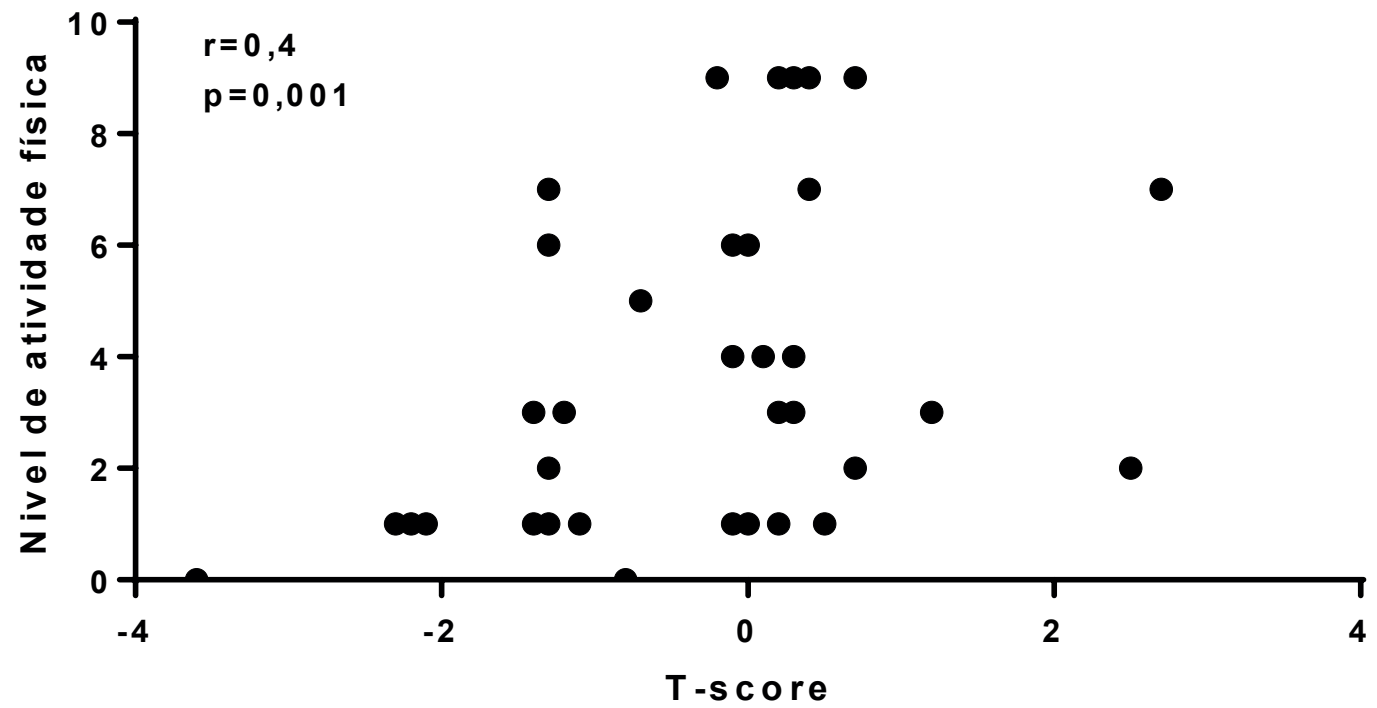

Figura 4. Correlação entre nível de atividade física (IPAC) com T-score.

\section{Correlação entre z-score e nível de atividade física}

Também foi encontrado uma correlação positiva entre o nível de atividade física e o Z-score, ou seja quanto maior foi o nível de atividade física que o indivíduo se encontrava, mais positivo foi o t-score, figura 5 


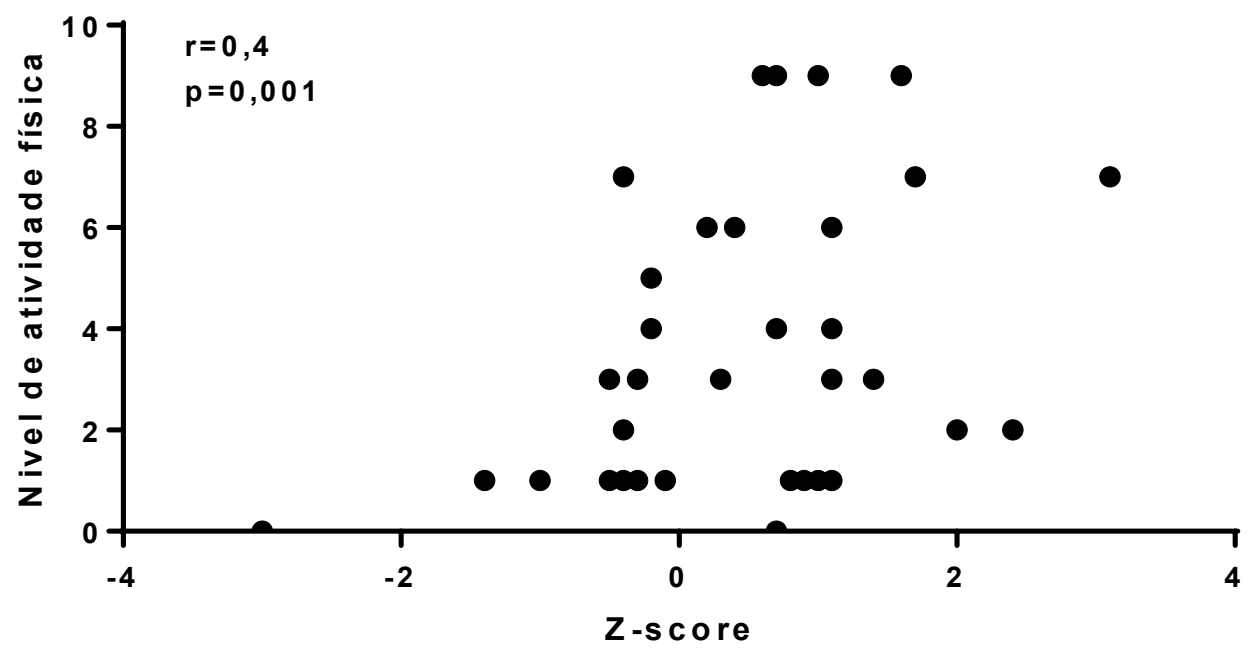

Figura 5. Correlação entre nível de atividade física (IPAC) e Z-score.

\section{DISCUSSÃO}

O presente estudo analisou a densidade mineral óssea em pacientes maiores de 40 anos, ativas fisicamente e sedentária. Dentre os principais resultados podem ser destacados que: pacientes que praticam exercícios regularmente, tem maior densidade mineral óssea em re- ta perda óssea relacionada à idade é provavelmente um decréscimo de formação óssea em nível celular, resultado da eficiência diminuída dos osteoblastos (MARINHO et al., 2014).

Além da idade, o sexo e a raça estão entre os principais determinantes da massa óssea e do risco de fraturas. Mulheres são mais suscetíveis à osteoporose do que homens, pois além de passarem pela menopausa também possuem menor DMO do que os homens. Fatores genéticos também são responsáveis pelas variações na massa óssea em diferentes grupos éticos e raciais. Indivíduos da raça negra possuem maior pico de massa óssea e, portanto, são menos predispostos a sofrerem de osteoporose que brancos e asiáticos (JERONYMO et al., 2012).

0 índice de massa corporal (IMC) está diretamente relacionado à DM0, sendo que os indivíduos obesos têm maior proteção contra osteoporose. Fatores ambientais como hábitos nutricionais, sedentarismo e tabagismo, além de doenças e drogas, também podem estar associados à redução da massa óssea (MARINHO et al., 2014).

Foi visto em nosso estudo que mulheres inativas fisicamente também tem maior propensão para perda de densidade mineral óssea. 0 exercício físico atua no osso por efeito direto, via força mecânica, ou indireto, mediado por fatores hormonais. Mas os mecanismos pelos quais a atividade física melhora a massa óssea ainda não são totalmente conhecidos. Em humanos, foi demonstrada redução de 0,9\% por semana do volume ósseo trabecular associado à diminuição de 1,3\% dia da força de contração muscular após imobilização prolongada 
(KNUTH et al., 2011, LORENTZON et al., 2015, CILLINAME et al., 2002).

Há indícios de que a atividade física minimiza a osteopenia decorrente do avançar da idade e do declínio dos esteroides sexuais. Contudo, não são todos os tipos de exercício que promovem efeito benéfico sobre o esqueleto de mulheres na pós-menopausa. Há estudos que demonstram que exercícios com carga de peso moderada como caminhadas, cooper, promovem aumento do conteúdo mineral nos ossos dessas mulheres. Ao contrário, foi observado em mulheres na pós-menopausa, submetidas a exercícios físicos de baixa carga de peso como natação, que são satisfatórios no condicionamento cardiovascular, mas não promovem alteração no conteúdo mineral do esqueleto (CHERIAN et al., 2003).

Além disso, também é comprovado que os exercícios físicos, com carga de peso moderada, ajudam na manutenção ou no ganho de massa óssea em mulheres na pós-menopausa (BECK et al., 2017). Nessa mesma fase, a atividade física quase sempre causa efeito anabólico sinérgico, quando associada a um tipo de tratamento convencional da osteoporose (OTERO et al. 2017). A atividade física promove alterações no metabolismo ósseo por efeito direto, via força mecânica, ou indireto, promovido por fatores hormonais (BERARD et al., 2097, MOSEKILDE et al.,1999). A força mecânica, quando aplicada sobre o tecido ósseo, forma sinais endógenos que interferem nos processos de remodelação óssea. Esses sinais são captados por um sistema mecanossensorial no qual o osteócito é a principal célula responsável por traduzir a força mecânica em sinais bioquímicos que regulam o turnover ósseo (PALERMO et al., 2015, BRAHM et al., 1997). Acredita-se que a deformação celular causada pela força direta sobre a célula, o aumento da pressão intracanalicular provocado pela força dinâmica e o incremento da velocidade do fluxo do fluido intersticial sejam fatores que afetam diretamente o osteócito, sendo esse último o maior estímulo ao osteócito em resposta à carga mecânica. 0 fluxo de fluido intersticial pelos canalículos ao redor do osteócito parece ser responsável pela deformação da matriz extracelular e por alterações nas membranas celulares (PALERMO et al., 2015).

Devido à existência de forte relação entre atividade física, densidade óssea mineral e massa óssea, a atividade física tem sido preconizada como estratégia para im- pedir a perda óssea e para manter a integridade esquelética (CHERIAN et al., 2003).

Outro fator importante associado a perda da densidade mineral óssea é o consumo diário de refrigerante e café, outros estudos mostram que o uso abusivo do café está associado ao risco de osteoporose ${ }^{3}$. Isto pode estar relacionado a química do refrigerante (NOMURA et al.,2000).

0 consumo de refrigerantes pode levar a efeitos adversos na densidade mineral óssea, porém estudos realizados até em agora adolescentes mostraram resultados inconclusos. As adolescentes do sexo feminino mostraram, por exemplo, que o alto consumo de refrigerantes levava a ocorrência de um maior número de fraturas e a baixa densidade mineral óssea; entretanto, não se sabe se esta ocorrência surge porque as meninas bebem menos leite, ou se haveria um efeito negativo do próprio refrigerante (MO et al., 2002).

Para investigar melhor esta situação, pesquisadores da Tufts University, de Boston, Estados Unidos, mediram a densidade mineral óssea (DMO) na coluna e na região coxofemoral em 1.413 mulheres e 1.125 homens. Os pesquisadores verificaram que ocorreu associação entre a ingestão de refrigerantes, de um modo geral, e a DMO. As mulheres que ingeriram mais refrigerantes à base de cola, tiveram uma significativa diminuição da densidade, em suas articulações coxofemorais 3.7\% e $5,4 \%$ de forma geral: quanto mais refrigerantes com cola as mulheres ingeriram, menos espessos eram os ossos. O consumo de refrigerantes com cola não teve efeito sobre os homens (MO et al., 2002).

Teoricamente, as dietas ricas em fósforo e pobre em cálcio podem levar a reduzir cálcio sérico, estimulando o hormônio paratireoide (PTH), o qual, por sua vez, faz com que ocorra reabsorção óssea e retornando o cálcio sérico a níveis normais. Apesar de ter sido sugerido que a quantidade de ácido fosfórico em cola é insuficiente para causar este desequilíbrio ${ }^{7}$, não fica claro se a exposição regular ao ácido fosfórico sem exposição ao cálcio ou outros nutrientes benéficos lentamente afeta a remodelação óssea e provoca a perda de osso ao longo do tempo. Entretanto já foi demonstrado que dietas ricas em fósforo provoca a perda óssea em animais (PORTO et al., 2017). Em um estudo, a cola foi dada a ratos imaturos e adultos e encontra-se que em ambos os grupos houve desenvolvidos hipercalciúria significativa e hi- 
perfosfatúria; os animais mais velhos também desenvolveu hiperparatireoidismo (KATHERINE et al., 2006). Em outro estudo, a cola foi dada a ratos ovariectomizados; foi observada hipocalcemia e perda de densidade mineral óssea femoral posterior em ratos em relação a um grupo de controlo (GAZZOTTI et al., 2019).

Outro fator possível relacionado, que causa redução da DMO é a quantidade de sacarose existente nos refrigerantes. Em um copo de $600 \mathrm{ml}$ de refrigerante tem em média 63 g de açúcar (NOMURA et al.,2000). 0 açúcar refinado é considerado uma caloria vazia, ela precisar de íons extras para ser processada, no caso da sacarose que a molécula essencialmente concentrada no açúcar refinado, ela necessita de cálcio, demandado do cálcio plasmático ${ }^{18}$, que é reposto por reabsorção óssea. Este modelo pode ser mais bem explicado pela ativação do sistema de sinalização RANK / RANKL / OPG que é essencial para a remodelação óssea. O Ligante de RANK (RANKL) é uma proteína de membrana do pré-osteoblásticas ou secretada por osteócitos, que se liga a RANK receptor de células precursoras de osteoclastos, promovendo, assim, a diferenciação e ativação de osteoclastos para a sua maturidade. Os osteoblastos, por sua vez, também segregam proteína OPG solúvel que atua como um receptor de engodo e interage com RANKL, que impede a ligação de RANK, inibi a osteoclastogénese. 0 equilíbrio entre OPG e RANKL e a ligação deste último ao seu receptor RANK é fundamental na determinação da anabólicos e catabólicos do osso. Assim, o gene TNFRSF11A (lócus 18q22.1), que codifica RANK, é particu- larmente importante na remodelação óssea, uma vez que determina a diferenciação de osteoclastos e sobrevivência. Uma alteração neste complexo causa uma desregulação de remodelação óssea pode causar condições patológicas, como seria a osteoporose (GARCIA-CONTRERAS et al., 2000, AMATO et al.,1998, CASTROGIOVANN et al.,1994) . A sacarose quando ingerida excessivamente pode ser responsável por esta alteração (CHERIAN et al., 2003).

Devido ao alto número de indivíduos que consomem refrigerante diariamente ou são considerados sedentários, é necessário que a população feminina seja alertada precocemente contra estes fatores que produzem redução da DMO e melhora do estilo de vida (PORTO et al., 2020)

As limitações deste estudo estão relacionadas ao fato de não termos controlado o tipo de exercício a frequência e intensidade para o grupo considerando ativo fisicamente. Devido a isto não podermos recomendar um tipo de exercício para evitar a perda de DMO, sugerimos que outros estudos sejam realizados para verificar os melhores métodos de treinamento capazes de prevenir osteoporose.

\section{CONCLUSÂO}

Em um estudo com mulheres sedentárias e ativas fisicamente pode-se concluir que o grupo de mulheres ativa fisicamente apresentaram estilo de vida mais positivo, maior densidade mineral óssea, consecutivamente menor risco para osteopenia e osteoporose. 


\section{REFERENCIAS}

AMATO, Dante et al. Acute effects of soft drink intake on calcium and phosphate metabolism in immature and adult rats. Revista de investigacion clinica; organo del Hospital de Enfermedades de la Nutricion, v. 50, n. 3, p. 185-189, 1998

BECK, Belinda R. et al. Exercise and Sports Science Australia (ESSA) position statement on exercise prescription for the prevention and management of osteoporosis. Journal of science and medicine in sport, v. 20, n. 5, p. 438-445, 2017.

BERARD, A.; BRAVO, G.; GAUTHIER, P. Meta-analysis of the effectiveness of physical activity for the prevention of bone loss in postmenopausal women. Osteoporosis international, v. 7 , n. 4, p. 331-337, 1997

BUTTROS, Davi de Araújo Brito et al . Risk factors for osteoporosis in postmenopausal women from southeast. Brazilian, $\mathrm{v}$. 33, n. 6, p. 295-302, June 2011

BRAHM, Helena et al. Net fluxes over working thigh of hormones, growth factors and biomarkers of bone metabolism during short lasting dynamic exercise. Calcified tissue international, v. 60 , n. 2, p. 175-180, 1997.

CAPUTO, Eduardo Lucia; COSTA, Marcelo Zanusso. Influence of physical activity on quality of life in postmenopausal women with osteoporosis. Rev. Bras. Reumatol., São Paulo, v. 54, n. 6 , p. 467-473, Dec. 2014.

CASTROGIOVANNI, Paola et al. The importance of physical activity in osteoporosis. From the molecular pathways to the clinical evidence. Histology and histopathology, v. 31, n. 11, p. 1183-1194, 2016.

CHERIAN, Priscilla P. et al. Effects of mechanical strain on the function of Gap junctions in osteocytes are mediated through the prostaglandin EP2 receptor. Journal of Biological Chemistry, v. 278, n. 44, p. 43146-43156, 2003.

CULLINANE, D. M. The role of osteocytes in bone regulation mineral homeostasis versus mechanoreception. Journal of Musculoskeletal and Neuronal Interactions, v. 2, n. 3, p. 242 244, 2002.

DALLANEZI, Glauber et al. Physical activity level of post-menopausal women with low bone mineral density. Revista Brasileira de Ginecologia e Obstetrícia/RBGO Gynecology and Obstetrics, v. 38, n. 05, p. 225-230, 2016.

GARCÍA-CONTRERAS, Fernando et al. Cola beverage consumption induces bone mineralization reduction in ovariectomized rats. Archives of Medical Research, v. 31, n. 4, p. 360-365 2000.

GAZZOTTI, Mariana R. et al. Frequency of Osteoporosis and Vertebral Fractures in Chronic Obstructive Pulmonary Disease
(COPD) Patients. Archivos de Bronconeumología (English Edition), v. 55, n. 5, p. 252-257, 2019.

JERONYMO, Lelia Paes; GARIBA, Munir Antonio. Lumbar spine digital radiography sensitivity and specifi city as a tool for the diagnosis of osteoporosis. Fisioter. mov., Curitiba, v. 25, n. 3, p. 607-615, Sept. 2012.

KNUTH, Alan Goularte et al. Practice of physical activity and sedentarism among Brazilians: results of the National Household Sample Survey-2008. Ciencia \& saude coletiva, v. 16, n. 9 , p. 3697,2011

KOHRT, Wendy M.; EHSANI, Ali A.; BIRGE JR, Stanley J. Effects of exercise involving predominantly either joint-reaction or ground-reaction forces on bone mineral density in older women. Journal of Bone and Mineral Research, v. 12, n. 8, p. 1253-1261, 1997.

LORENTZON, Mattias; CUMMINGS, Steven R. Osteoporosis: the evolution of a diagnosis. Journal of internal medicine, v. 277, n. 6, p. 650-661, 2015 .

MARINHO, Bruna Coelho Galvão et al . The burden of osteoporosis in Brazil. Arq Bras Endocrinol Metab, São Paulo, v. 58, n. 5, p. 434-443, July 2014.

MOSEKILDE, Li et al. Additive effect of voluntary exercise and growth hormone treatment on bone strength assessed at four different skeletal sites in an aged rat model. Bone, v. 24, n. 2, p. 71-80, 1999.

MOREIRA, Linda Denise Fernandes et al. Physical exercise and osteoporosis: effects of different types of exercises on bone and physical function of postmenopausal women. Arquivos Brasileiros de Endocrinologia \& Metabologia, v. 58, n. 5, p. 514$522,2014$.

MO, A. et al. Bipedal stance exercise and prostaglandin E2 (PGE2) and its synergistic effect in increasing bone mass and in lowering the PGE2 dose required to prevent ovariectomized-induced cancellous bone loss in aged rats. Bone, v. 31, n. 3, p. 402-406, 2002.

NOMURA, Shintaro; TAKANO-YAMAMOTO, Teruko. Molecular events caused by mechanical stress in bone. Matrix Biology, v. 19, n. 2, p. 91-96, 2000.

OTERO, Montserrat et al. The effectiveness of a basic exercise intervention to improve strength and balance in women with osteoporosis. Clinical interventions in aging, v. 12, p. 505, 2017.

PALERMO, Andrea et al. Irisin is associated with osteoporotic fractures independently of bone mineral density, body composition or daily physical activity. Clinical endocrinology, v. 82, n. 4, p. 615-619, 2015 
PORTO, E. F., OLIVEIRA, F. C., DA ROCHA, G. P. F., de Aquiles Bernardo, K. M., Kumpel, C., \& de Castro, A. A. M. Lifestyle and its correlations with osteoporosis and loss of body balance. Revista Família, Ciclos de Vida e Saúde no Contexto Social, v. 5, n. 2, p. 200-208, 2017.

PORTO, E., DE OLIVEIRA PIMENTEL, T., BERNARDES, F. A., CAMARGO, A. C., \& KUMPEL, C. Efeitos da atividade física inespecífica sobre o equilíbrio corporal em idosos. Life Style, v. 6, n. 2, p. 73-82, 2019.
SOUZA, Márcio Passini Gonçalves de. Osteoporosis diagnosis and treatment. Rev. bras. ortop., São Paulo, v. 45, n. 3, p. 220229, June 2010.

TUCKER, Katherine L. et al. Colas, but not other carbonated beverages, are associated with low bone mineral density in older women: The Framingham Osteoporosis Study. The American journal of clinical nutrition, v. 84, n. 4, p. 936 942, 2006 